This item was submitted to Loughborough's Institutional Repository (https://dspace.lboro.ac.uk/) by the author and is made available under the following Creative Commons Licence conditions.

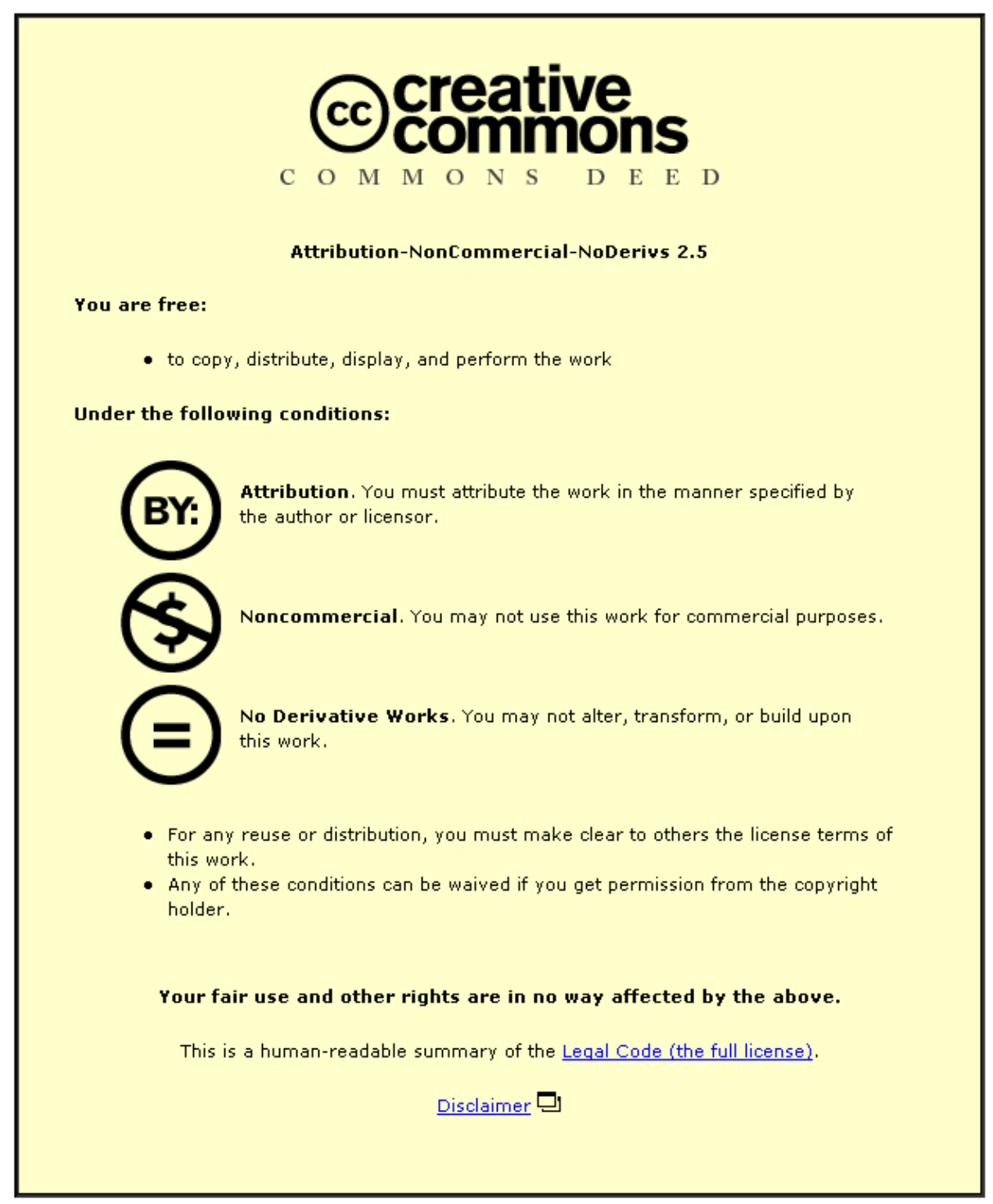

For the full text of this licence, please go to: http://creativecommons.org/licenses/by-nc-nd/2.5/ 


\title{
Thermography based breast cancer analysis using statistical features and fuzzy classification
}

\author{
Gerald Schaefer $^{1}$, Michal Zavisek ${ }^{2}$ and Tomoharu Nakashima ${ }^{3}$
}

\author{
${ }^{1}$ School of Engineering and Applied Science, \\ Aston University, U.K. \\ ${ }^{2}$ Faculty of Electrical Engineering and Communication, \\ Brno University of Technology, Czech Republic \\ ${ }^{3}$ Department of Computer Science and Intelligent Systems, \\ Osaka Prefecture University, Japan
}

\begin{abstract}
Medical thermography has proved to be useful in various medical applications including the detection of breast cancer where it is able to identify the local temperature increase caused by the high metabolic activity of cancer cells. It has been shown to be particularly well suited for picking up tumors in their early stages or tumors in dense tissue and outperforms other modalities such as mammography for these cases. In this paper we perform breast cancer analysis based on thermography, using a series of statistical features extracted from the thermograms quantifying the bilateral differences between left and right breast areas, coupled with a fuzzy rule-based classification system for diagnosis. Experimental results on a large dataset of nearly 150 cases confirm the efficacy of our approach that provides a classification accuracy of about $80 \%$.
\end{abstract}


Keywords: cancer diagnosis, breast cancer, medical thermography, image analysis, pattern classification

\section{Introduction}

Advances in camera technologies and reduced equipment costs have lead to an increased interest in the application of thermography in the medical field [11]. Thermal medical imaging (or medical infrared imaging) uses a camera with sensitivities in the infrared to provide a picture of the temperature distribution of the human body or parts thereof. It is a non-invasive, non-contact, passive, radiation-free technique that can also be used in combination with anatomical investigations based on x-rays and three-dimensional scanning techniques such as CT and MRI and often reveals problems when the anatomy is otherwise normal. It is well known that the radiance from human skin is an exponential function of the surface temperature which in turn is influenced by the level of blood perfusion in the skin. Thermal imaging is hence well suited to pick up changes in blood perfusion which might occur due to inflammation, angiogenesis or other causes. Asymmetrical temperature distributions as well as the presence of hot and cold spots are known to be strong indicators of an underlying dysfunction [17].

Breast cancer is the most commonly diagnosed form of cancer in women accounting for about $30 \%$ of all cases [1]. Despite earlier, less encouraging studies, which were based on low-capability and poorly calibrated equipment, infrared imaging has been shown to be well suited for the task of detecting breast cancer, in particular when the tumor is in its early stages or in dense tissue $[2,6]$. Early detection is important as it provides significantly higher chances of survival [3] and in this respect infrared imaging outperforms the standard method of mammography which can detect tumors only 
once they exceed a certain size. On the other hand, tumors that are small in size can be identified using thermography ${ }^{1}$ due to the high metabolic activity of cancer cells which leads to an increase in local temperature that can be picked up in the infrared.

In this paper we perform breast cancer analysis based on thermography, using a series of statistical features extracted from the thermograms coupled with a fuzzy rule-based classification system for diagnosis. The features stem from a comparison of left and right breast areas and quantify the bilateral differences encountered. Following this asymmetry analysis the features are fed to a fuzzy classification system. This classifier is used to extract fuzzy if-then rules based on a training set of known cases. Experimental results on a set of nearly 150 cases show the proposed system to work well accurately classifying about $80 \%$ of cases, a performance that is comparable to other imaging modalities such as mammography.

The rest of the paper is organised as follows: The following section covers the features we extract from the breast thermograms. Section 3 introduces the fuzzy rule-based classifier we employ while Section 4 presents experimental results obtained. Section 5 concludes the paper.

\section{Breast thermogram feature analysis}

Thermograms for breast cancer diagnosis are usually taken based on a frontal view and/or some lateral views. In our work we restrict out attention to frontal view images. As has been shown earlier, an effective approach to automatically detect cancer cases is to study the symmetry between the left and right breast [14]. In the case of cancer presence the tumor will re-

\footnotetext{
${ }^{1}$ according to [12] the average tumor size undetected by mammography is $1.66 \mathrm{~cm}$ compared to only $1.28 \mathrm{~cm}$ by thermography
} 
cruit blood vessels resulting in hot spots and a change in vascular pattern, and hence an asymmetry between the temperature distributions of the two breast. On the other hand, symmetry typically identifies healthy subjects. We therefore follow this approach and segment the areas corresponding to the left and right breast from the thermograms. While some advances have been made in trying to automatically segment breast regions from thermograms [13] we found that they are not robust enough yet to account for the variety of cases present in our dataset. In our work, the regions corresponding to the left and right breast were therefore manually segmented by a medical expert. Once segmented, we convert the breast regions to a polar co-ordinates representation as it simplifies the calculation of several of the features that we employ. A series of statistical features is then calculated to provide indications of symmetry between the regions of interest (i.e. the two breasts). In the following sections we describe the features we employ.

\subsection{Basic statistical features}

Clearly the simplest feature to describe a temperature distribution such as those encountered in thermograms is to calculate its statistical mean. As we are interested in symmetry features we calculate the mean for both breasts and use the absolute value of the difference of the two. Similarly we calculate the standard temperature deviation and use the absolute difference as a feature. Furthermore we employ the absolute differences of the median temperature and the 90-percentile as further descriptors. 


\subsection{Moments}

Image moments are defined as

$$
m_{p q}=\sum_{y=0}^{M-1} \sum_{x=0}^{N-1} x^{p} y^{q} g(x, y)
$$

where $x$ and $y$ define the pixel location and $N$ and $M$ the image size. We utilise moments $m_{01}$ and $m_{10}$ which essentially describe the centre of gravity of the breast regions, as well as the distance (both in $x$ and $y$ direction) of the centre of gravity from the geometrical centre of the breast. For all four features we calculate the absolute differences of the values between left and right breast.

\subsection{Histogram features}

Histograms record the frequencies of certain temperature ranges of the thermograms. In our work we construct normalised histograms for both regions of interest (i.e. left and right breast) and use the cross-correlation between the two histograms as a features. From the difference histogram (i.e. the difference between the two histograms) we compute the absolute value of its maximum, the number of bins exceeding a certain threshold (empirically set to 0.01 in our experiments), the number of zero crossings, energy and the difference of the positive and negative parts of the histogram.

\subsection{Cross co-occurrence matrix}

Co-occurrence matrices have been widely used in texture recognition tasks [5] and can be defined as

$$
\gamma_{T_{i}, T_{j}}^{(k)}(I)=\mathrm{PR}_{p_{1} \in I_{T_{i}}, p_{2} \in I}\left[p_{2} \in I_{T_{j}},\left|p_{1}-p_{2}\right|=k\right]
$$


with

$$
\left|p_{1}-p_{2}\right|=\max \left|x_{1}-x_{2}\right|,\left|y_{1}-y_{2}\right|
$$

where $T_{i}$ and $T_{j}$ denote two temperature values and $\left(x_{k}, y_{k}\right)$ denote pixel locations. In other words, given any temperature $T_{i}$ in the thermogram, $\gamma$ gives the probability that a pixel at distance $k$ away is of temperature $T_{j}$. In order to arrive at an indication of asymmetry between the two sides we adopted this concept and derived what we call a cross co-occurrence matrix defined as

$$
\gamma_{T_{i}, T_{j}}^{(k)}(I(1), I(2))=\mathrm{PR}_{p_{1} \in I(1)_{T_{i}}, p_{2} \in I(2)}\left[p_{2} \in I(2)_{T_{j}},\left|p_{1}-p_{2}\right|=k\right]
$$

i.e. temperature values from one breast are related to temperatures of the second side.

From this matrix we can extract several features [5]. the ones we are using are

$$
\begin{aligned}
\text { Homogeneity } G & =\sum_{k} \sum_{l} \frac{\gamma_{k, l}}{1+|k-l|} \\
\text { Energy } E & =\sum_{k} \sum_{l} \gamma_{k, l}^{2} \\
\text { Contrast } C & =\sum_{k} \sum_{l}|k-l| \gamma_{k, l}
\end{aligned}
$$

and

$$
\text { Symmetry } S=1-\sum_{k} \sum_{l}\left|\gamma_{k, l}-\gamma_{l, k}\right|
$$

We further calculate the first four moments $m_{1}$ to $m_{4}$ of the matrix

$$
m_{p}=\sum_{k} \sum_{l}(k-l)^{p} \gamma_{k, l}
$$




\subsection{Mutual information}

The mutual information $M I$ between two distributions can be calculated from the joint entropy $H$ of the distributions and is defined as

$$
M I=H_{L}+H_{R}+H
$$

with

$$
\begin{aligned}
H_{L} & =-\sum_{k} P_{L}(k) \log _{2} p_{L}(k) \\
H_{R} & =-\sum_{l} P_{R}(l) \log _{2} p_{R}(l) \\
H & =\sum_{k} \sum_{l} P_{L R}(k, l) \log _{2} p_{L, R}(k, l)
\end{aligned}
$$

and

$$
\begin{aligned}
p_{L R}(k, l) & =x_{k, l} / \sum_{k, l} x(k, l) \\
p_{L}(k) & =\sum_{l} p_{L R}(k, l) \\
p_{R}(k) & =\sum_{k} p_{L R}(k, l)
\end{aligned}
$$

and is employed as a further descriptor.

\subsection{Fourier analysis}

As last feature descriptors we calculate the Fourier spectrum and use the difference of absolute values of the ROI spectra. The features we adopt are the difference maximum and the distance of this maximum from the centre. 


\subsection{Summary}

To summarise we characterise each breast thermogram using the following set of features: 4 basic statisical features, 4 moment features, 8 histogram features, 8 cross co-occurrence features, mutual information and 2 Fourier descriptors. We further apply a Laplacian filter to enhance the contrast and calculate another subset of features (the 8 cross co-oocurance features together with mutual information and the 2 Fourier descriptors) from the resulting images. In total we hence end up with 38 descriptors per breast thermogram which describe the asymmetry between the two sides. We normalise each feature to the interval $[0 ; 1]$ to arrive at comparable units between descriptors.

\section{Fuzzy rule-based classification}

While in the past fuzzy rule-based systems have been applied mainly to control problems [16], more recently they have also been applied to pattern classification problems. Various methods have been proposed for the automatic generation of fuzzy if-then rules from numerical data for pattern classification $[4,8,7]$ and have been shown to work well on a variety of problem domains.

Pattern classification typically is a supervised process where, based on set of training samples that have been manually classified by experts, a classifier is derived that automatically assigns unseen data sample to the pre-defined classes. Let us assume that our pattern classification problem is an $n$-dimensional problem with $C$ classes (in clinical diagnosis such as the detection of breast cancer $C$ is typically 2 ) and $m$ given training patterns $\mathbf{x}_{p}=\left(x_{p 1}, x_{p 2}, \ldots, x_{p n}\right), p=1,2, \ldots, m$. Without loss of generality, we 
assume each attribute of the given training patterns to be normalised into the unit interval $[0,1]$; that is, the pattern space is an $n$-dimensional unit hypercube $[0,1]^{n}$. In this study we use fuzzy if-then rules of the following type as a base of our fuzzy rule-based classification systems:

$$
\begin{aligned}
& \text { Rule } R_{j} \text { : If } x_{1} \text { is } A_{j 1} \text { and } \ldots \text { and } x_{n} \text { is } A_{j n} \\
& \text { then Class } C_{j} \text { with } C F_{j}, \quad j=1,2, \ldots, N \text {, }
\end{aligned}
$$

where $R_{j}$ is the label of the $j$-th fuzzy if-then rule, $A_{j 1}, \ldots, A_{j n}$ are antecedent fuzzy sets on the unit interval $[0,1], C_{j}$ is the consequent class (i.e. one of the $C$ given classes), and $C F_{j}$ is the grade of certainty of the fuzzy if-then rule $R_{j}$. As antecedent fuzzy sets we use triangular fuzzy sets as in Figure 1 where we show a partition of the unit interval into a number of fuzzy sets.

Our fuzzy rule-based classification system consists of $N$ fuzzy if-then rules each of which has a form as in Equation (13). There are two steps in the generation of fuzzy if-then rules: specification of antecedent part and determination of consequent class $C_{j}$ and the grade of certainty $C F_{j}$. The antecedent part of fuzzy if-then rules is specified manually. Then the consequent part (i.e. consequent class and the grade of certainty) is determined from the given training patterns [10]. In [9] it is shown that the use of the grade of certainty in fuzzy if-then rules allows us to generate comprehensible fuzzy rule-based classification systems with high classification performance.

\subsection{Fuzzy Rule Generation}

Let us assume that $m$ training patterns $\mathbf{x}_{p}=\left(x_{p 1}, \ldots, x_{p n}\right), p=1, \ldots, m$, are given for an $n$-dimensional $C$-class pattern classification problem. The consequent class $C_{j}$ and the grade of certainty $C F_{j}$ of the if-then rule are 
determined in the following two steps:

1. Calculate $\beta_{\text {Class } h}(j)$ for Class $h$ as

$$
\beta_{\text {Class } h}(j)=\sum_{\mathbf{x}_{p} \in \text { Class } h} \mu_{j}\left(\mathbf{x}_{p}\right),
$$

where

$$
\mu_{j}\left(\mathbf{x}_{p}\right)=\mu_{j 1}\left(x_{p 1}\right) \cdot \ldots \cdot \mu_{j n}\left(x_{p n}\right),
$$

and $\mu_{j n}(\cdot)$ is the membership function of the fuzzy set $A_{j n}$. In this chapter we use triangular fuzzy sets as in Figure 1.

2. Find Class $\hat{h}$ that has the maximum value of $\beta_{\text {Class } h}(j)$ :

$$
\beta_{\text {Class } \hat{h}}(j)=\max _{1 \leq k \leq C}\left\{\beta_{\text {Class } k}(j)\right\}
$$

If two or more classes take the maximum value, the consequent class $C_{j}$ of the rule $R_{j}$ can not be determined uniquely. In this case, specify $C_{j}$ as $C_{j}=\phi$. If a single class $\hat{h}$ takes the maximum value, let $C_{j}$ be Class $\hat{h}$. The grade of certainty $C F_{j}$ is determined as

$$
C F_{j}=\frac{\beta_{\text {Class } \hat{h}}(j)-\bar{\beta}}{\sum_{h} \beta_{\text {Class } h}(j)}
$$

with

$$
\bar{\beta}=\frac{\sum_{h \neq \hat{h}} \beta_{\text {Class } h}(j)}{C-1} .
$$

\subsection{Fuzzy Reasoning}

Using the rule generation procedure outlined above we can generate $N$ fuzzy if-then rules as in Equation (13). After both the consequent class $C_{j}$ and 
the grade of certainty $C F_{j}$ are determined for all $N$ rules, a new pattern $\mathbf{x}=\left(x_{1}, \ldots, x_{n}\right)$ can be classified by the following procedure:

1. Calculate $\alpha_{\text {Class } h}(\mathbf{x})$ for Class $h, j=1, \ldots, C$, as

$$
\alpha_{\text {Class } h}(\mathbf{x})=\max \left\{\mu_{j}(\mathbf{x}) \cdot C F_{j} \mid C_{j}=h\right\}
$$

2. Find Class $h^{\prime}$ that has the maximum value of $\alpha_{\text {Class } h}(\mathbf{x})$ :

$$
\alpha_{\text {Class } h^{\prime}}(\mathbf{x})=\max _{1 \leq k \leq C}\left\{\alpha_{\text {Class } k}(\mathbf{x})\right\}
$$

If two or more classes take the maximum value, then the classification of $\mathbf{x}$ is rejected (i.e. $\mathbf{x}$ is left as an unclassifiable pattern), otherwise we assign $\mathbf{x}$ to Class $h^{\prime}$.

\subsection{Rule reduction}

It is generally know that any type of rule-based system suffers from the course of dimensionality. That is, the number of generated rules exponentially increases with the number of attributes involved. Our fuzzy rule-based classifier is no exception, in particular considering the variety of features we are using as input. For example, based on the selection of the 38 features we employ, the classifier would generate $2^{38}=2.75 * 10^{11}$ rules even if we only partition each axis into two which is clearly prohibitive both in terms of storage requirements and computational complexity. In our approach we employ a genetic algorithm that evolves to select a fixed, small, number of rules (100 in our experiments) without sacrificing classification performance [7]. We also apply a cost term in the classification rules to be able to put more emphasis on correctly identifying maligant cases [15]. 


\section{Experimental results}

For our experiment we utilised a dataset of 146 thermograms (29 malignant and 117 benign cases). It should be noted that this dataset is significantly larger than those used in previous studies (e.g. [14]). For all thermograms we calculate a feature vector of length 38 as outlined in Section 2. We then train the fuzzy classifier explained in the previous section using this data to obtain a classifier that is capable of distinguishing cancer patients from healthy individuals.

As a first test we wish to examine how well the classifier is able to separate the two classes. We therefore train the classification system on all available data (i.e. on all 146 cases) and then test it on all samples. That is, for this experiment the training and test data are identical. We experiment with different number of fuzzy partitions per attribute. Preliminary experiments showed that fuzzy classifiers with less than 10 divisions per attribute were not sufficiently complex to handle the data at hand [15]. On the other hand, as explained above, finer partitioning of the attribute data results in a wider search space and hence a computational expensive derivation of the classifier. We therefore restrict our attention to classifiers with between 10 and 15 partitions per attribute.

Table 1 shows the results in terms of classification rate (i.e. the percentage of correctly classified patterns), sensitivity (i.e. the probability that a case identified as malignant is indeed malignant) and specificity (i.e. the probability that a case identified as benign is indeed benign). Looking at results we can see that classification performance lies roughly between 92 and $98 \%$ with the best performance, a sensitivity of about $93 \%$ coupled with a specificity of about $98 \%$ being achieved with 15 partitions. We notice that even though the classifiers are tested on the same data that was used for 
training we do not achieve perfect classification. This suggests that we have indeed a challenging data set to deal with as the two classes cannot even be separated by the non-linear division our fuzzy classifier is capable of.

While results on training data provides us with some basic indication of the classification performance, only validation on unseen test data will provide real insights into the generalisation capabilities of a classifier as normally classification accuracy on such unseen patterns is lower than that achieved in previously encountered training samples. We therefore perform standard 10-fold cross-validation on the dataset where the patterns are split into 10 disjoint sets and the classification performance of one such set based on training the classifier with the remaining $90 \%$ of samples evaluated in turn for all 10 combinations. Again, we explore classifiers with between 10 and 15 partition per attribute.

The results are listed in Table 2. From there we can see that - as expected - classification rates are lower than the ones we achieved on training data, with the best results just below $80 \%$ providing both a sensitivity and specificity of almost $80 \%$. That there is a fairly significant drop in terms of classification performance once again confirms the difficulty of the dataset. However it should be noted that a correct classification rate of $80 \%$ is comparable to that achieved by other techniques for breast cancer diagnosis with mammography typically providing about $80 \%$, ultrasonography about $70 \%$, MRI systems about $75 \%$ and DOBI (optical systems) reaching about $80 \%$ diagnostic accuracy [18]. We can therefore conclude that our presented approach is indeed useful as an aid for diagnosis of breast cancer and should prove even more powerful when coupled with another modality such as mammography. We want to stress that it is indeed as part of such A combination that we see the primary use of thermography in breast cancer analysis rather 
than conducting cancer diagnosis based solely on thermal imaging as some other researchers suggest.

\section{Conclusions}

In this paper we presented a computational approach to the diagnosis of breast cancer based on medical infrared imaging. Asymmetry analysis of breast thermograms is performed using a variety of statistical features. These features are then fed into a fuzzy if-then rule based classification system which outputs a diagnostic prediction of the investigated patient. Experimental results on a large dataset of thermograms confirm the efficacy of the approach providing a classification accuracy of about $80 \%$ which is comparable to the performance achieved by other techniques including mammography.

\section{References}

[1] American Cancer Society. Cancer facts and figures. http://www . cancer.org/docroot/STT/stt_0.asp.

[2] N. Anbar, L. Milescu, A. Naumov, C. Brown, T. Button, C. Carly, and K. AlDulaimi. Detection of cancerous breasts by dynamic area telethermometry. IEEE Engineering in Medicine and Biology Magazine, 20(5):80-91, 2001.

[3] M. Gautherie. Thermobiological assessment of benign and maligant breast diseases. Am. J. Obstet. Gynecol., 147(8):861-869, 1983. 
[4] M. Grabisch and F. Dispot. A comparison of some methods of fuzzy classification on real data. In 2nd Int. Conference on Fuzzy Logic and Neural Networks, pages 659-662, 1992.

[5] R.M. Haralick. Statistical and structural approaches to texture. Proceedings of the IEEE, 67(5):786-804, May 1979.

[6] J.F. Head, F. Wang, C.A. Lipari, and R.L. Elliott. The important role of infrared imaging in breast cancer. IEEE Engineering in Medicine and Biology Magazine, 19:52-57, 2000.

[7] H. Ishibuchi and T. Nakashima. Improving the performance of fuzzy classifier systems for pattern classification problems with continuous attributes. IEEE Trans. on Industrial Electronics, 46(6):1057-1068, 1999.

[8] H. Ishibuchi and T. Nakashima. Performance evaluation of fuzzy classifier systems for multi-dimensional pattern classification problems. IEEE Trans. Systems, Man and Cybernetics - Part B: Cybernetics, 29:601618, 1999.

[9] H. Ishibuchi and T. Nakashima. Effect of rule weights in fuzzy rulebased classification systems. IEEE Trans. Fuzzy Systems, 9(4):506-515, 2001.

[10] H. Ishibuchi, K. Nozaki, and H. Tanaka. Distributed representation of fuzzy rules and its application to pattern classification. Fuzzy Sets and Systems, 52(1):21-32, 1992.

[11] B.F. Jones. A reappraisal of infrared thermal image analysis for medicine. IEEE Trans. Medical Imaging, 17(6):1019-1027, 1998. 
[12] J.R. Keyserlingk, P.D. Ahlgren, E. Yu, N. Belliveau, and M. Yassa. Functional infrared imaging of the breast. IEEE Engineering in Medicine and Biology Magazine, 19(3):30-41, 2000.

[13] H. Qi and J. F. Head. Asymmetry analysis using automatic segmentation and classification for breast cancer detection in thermograms. In 23rd IEEE Int. Conference on Engineering in Medicine and Biology, 2001.

[14] H. Qi, W. E. Snyder, J. F. Head, and R. L. Elliott. Detecting breast cancer from infrared images by asymmetry analysis. In 22nd IEEE Int. Conference on Engineering in Medicine and Biology, 2000.

[15] G. Schaefer, T. Nakashima, M. Zavisek, A. Drastich, and Y. Yokota. Cost-sensitive classification of breast cancer thermograms. Thermology International, 16(3), 2006.

[16] M. Sugeno. An introductory survey of fuzzy control. Information Science, 30(1/2):59-83, 1985.

[17] S. Uematsu. Symmetry of skin temperature comparing one side of the body to the other. Thermology, 1(1):4-7, 1985.

[18] M. Zavisek and A. Drastich. Thermogram classification in breast cancer detection. In 3rd European Medical and Biological Engineering Conference, pages 1727-1983, 2005. 


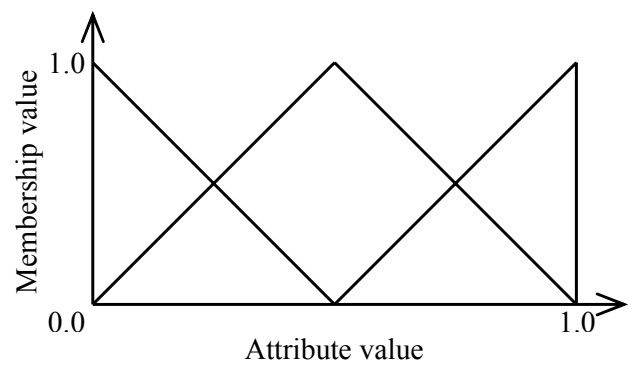

Figure 1: Membership function. 


\begin{tabular}{c|c|c|c} 
\# fuzzy partitions & classification rate [\%] & sensitivity [\%] & specificity [\%] \\
\hline \hline 10 & 91.78 & 82.76 & 94.02 \\
\hline 11 & 92.47 & 82.76 & 96.58 \\
\hline 12 & 92.47 & 86.21 & 95.73 \\
\hline 13 & 97.26 & 93.10 & 98.30 \\
\hline 14 & 94.52 & 89.66 & 95.73 \\
\hline 15 & 97.95 & 93.10 & 99.15
\end{tabular}

Table 1: Results of breast cancer thermogram classification on training data. 


\begin{tabular}{c|c|c|c} 
\# fuzzy partitions & classification rate [\%] & sensitivity [\%] & specificity [\%] \\
\hline \hline 10 & 78.05 & 74.14 & 79.02 \\
\hline 11 & 76.57 & 72.41 & 77.60 \\
\hline 12 & 77.33 & 75.52 & 77.78 \\
\hline 13 & 78.05 & 77.42 & 78.21 \\
\hline 14 & 79.53 & 79.86 & 79.49 \\
\hline 15 & 77.43 & 76.00 & 77.78
\end{tabular}

Table 2: Results of breast cancer thermogram classification on test data based on 10-fold cross validation. 